\title{
A Note on Solitons with Generalized Geodesic Vector Field
}

\author{
Adara M. Blaga ${ }^{1,+} \mathbb{D}$, Amira Ishan ${ }^{2,+}\left(\mathbb{D}\right.$ and Sharief Deshmukh ${ }^{3, *,+} \mathbb{D}$ \\ 1 Department of Mathematics, West University of Timisoara, Bd. V. Parvan, No. 4, 300223 Timisoara, Romania; \\ adara.blaga@e-uvt.ro \\ 2 Department of Mathematics, College of Science, Taif University, P.O. Box 11099, Taif 21944, Saudi Arabia; \\ a.ishan@tu.edu.sa \\ 3 Department of Mathematics, College of Science, King Saud University, P.O. Box 2455, \\ Riyadh 11451, Saudi Arabia \\ * Correspondence: shariefd@ksu.edu.sa \\ + These authors contributed equally to this work.
}

check for

updates

Citation: Blaga, A.M.; Ishan, A.; Deshmukh, S. A Note on Solitons with Generalized Geodesic Vector Field. Symmetry 2021, 13, 1104. https://doi.org/10.3390/sym13071104

Academic Editor: Youssef N. Raffoul

Received: 8 May 2021

Accepted: 15 June 2021

Published: 22 June 2021

Publisher's Note: MDPI stays neutral with regard to jurisdictional claims in published maps and institutional affiliations.

Copyright: (C) 2021 by the authors. Licensee MDPI, Basel, Switzerland. This article is an open access article distributed under the terms and conditions of the Creative Commons Attribution (CC BY) license (https:/ / creativecommons.org/licenses/by/ $4.0 /)$.
Abstract: We consider a general notion of an almost Ricci soliton and establish some curvature properties for the case in which the potential vector field of the soliton is a generalized geodesic or a 2-Killing vector field. In this vein, we characterize trivial generalized Ricci solitons.

Keywords: Ricci soliton; generalized geodesic vector field

MSC: 53C15; 53C65

\section{Introduction}

Self-similar solutions of the well-known geometric flows Ricci, Einstein and Yamabe solitons can be naturally extended on a Riemannian manifold to a more general notion of soliton, which we further consider. Precisely, if there exist a vector field $\xi$ and two smooth functions $\alpha$ and $\beta$ on the Riemannian manifold $(M, g)$ such that the Ricci curvature of $g$ satisfies

$$
\frac{1}{2} £_{\xi} g+\alpha \operatorname{Ric}=\beta g,
$$

then $(g, \xi, \alpha, \beta)$ defines a generalized Ricci soliton. Note that if $(g, \xi, \lambda)$ is an almost Ricci soliton [1], then $(\alpha, \beta)=(1, \lambda)$, and if $(g, \xi, \lambda)$ is an almost Riemann soliton [2], with $n>2$, then $(\alpha, \beta)=\left(\frac{1}{n-2}, \frac{(n-1) \lambda-\operatorname{div}(\xi)}{n-2}\right)$.

In particular, if the potential vector field $\xi$ of the soliton is a Killing vector field, i.e., $£_{\xi} g=0$, then we call the soliton trivial. Killing vector fields on a Riemannian manifold have been generalized to 2-Killing vector fields defined as those vector fields satisfying the weaker condition $£_{\xi} £_{\xi} g=0$. Such 2-Killing vector fields were firstly considered by Németh in [3], their importance in Lorentzian geometry being underlined by Cruz Neto, Melo and Sousa in [4]. Of course, all Killing vector fields are 2-Killing, but there are also examples of 2-Killing vector fields that are not Killing; we refer the reader to [3] for more information on these. It is therefore natural to seek the restrictions on the potential vector field $\xi$ to be 2-Killing and identify conditions under which a generalized Ricci soliton on a Riemannian manifold is trivial.

On the other hand, extending the notion of the unit geodesic vector field, the generalized geodesic vector fields [5] are the those for which the integral curves have accelerated velocity.

Examples of the generalized geodesic vector field are provided by the position vector field in the Euclidean space, the potential vector field of a gradient Yamabe soliton $[5,6]$, vector fields appearing in an Eikonal equation [7] etc. The class of generalized geodesic vector fields contains the concircular vector fields [8] that have applications in general relativity. Remark that in [6], the authors characterize the $n$-spheres by means of generalized geodesic vector fields. Among special vector fields, unit geodesic vector fields and 
generalized geodesic vector fields are the simplest vector fields, and a Killing vector field is a generalized geodesic vector field, but the opposite is not true. For examples of generalized geodesic vector fields that are not Killing vector fields, we refer the reader to $[5,6]$. Therefore, another natural question regards restricting the potential vector field $\xi$ to be a generalized geodesic vector field and identifying conditions under which a generalized Ricci soliton on a Riemannian manifold is trivial.

The aim of this paper is to provide some sufficient conditions for a generalized Ricci soliton on a Riemannian manifold to be trivial. Precisely, if the potential vector field of a generalized Ricci soliton is a generalized geodesic or a 2-Killing vector field, we provide conditions for it to be a Killing vector field, i.e., for the soliton to be trivial.

\section{Solitons with Generalized Geodesic Vector Field}

In this section, we further consider the definition provided below:

Definition 1. We say that $(g, \xi, \alpha, \beta)$ is a generalized Ricci soliton on an $n$-dimensional smooth manifold $M(n>2)$ if the Riemannian metric $g$, the vector field $\xi$ and the smooth functions $\alpha$ and $\beta$ satisfy

$$
\frac{1}{2} £_{\xi} g+\alpha \text { Ric }=\beta g,
$$

where $£_{\xi} g$ is the Lie derivative in the direction of $\xi$, and Ric is the Ricci curvature of $g$.

Some examples of nontrivial generalized Ricci solitons are as follows:

Example 1. Let $\xi$ be a nontrivial conformal vector field on an n-dimensional Einstein manifold $(M, g)$. Then, we have

$$
£_{\xi} g=2 \rho g, \quad \text { Ric }=\frac{r}{n} g
$$

and consequently, for a smooth function $\alpha$ on $M$, we have

$$
\frac{1}{2} £_{\xi} g+\alpha \text { Ric }=\beta g,
$$

where $\beta=\rho+\frac{1}{n} \alpha$. Thus, $(g, \xi, \alpha, \beta)$ is a generalized Ricci soliton on $M$.

Example 2. Consider the $n$-sphere $\mathbf{S}^{n}(c)$ of constant curvature $c$. Then, we have Ric $=(n-1) c g$, and there exists a non-parallel closed vector field $\xi$ on $\mathbf{S}^{n}(c)$ that satisfies

$$
£_{\xi} g=2 \rho g,
$$

where $\rho$ is a non-constant function such that

$$
\int_{S^{n}(c)} \rho=0 .
$$

Furthermore, for a smooth function $\alpha$ on $\mathbf{S}^{n}(c)$, we have

$$
\frac{1}{2} £_{\xi} g+\alpha \text { Ric }=\beta g,
$$

where $\beta=\rho+(n-1) c \alpha$. Thus, $(g, \xi, \alpha, \beta)$ is a generalized Ricci soliton on $\mathbf{S}^{n}(c)$.

Example 3. Let $(M, g)$ be an $n$-dimensional Riemannian manifold, and let $\rho$ be a smooth function on $M$. Then, for a real number $\tau, 0<\tau \leq \infty$, the triple $(M, g, \rho)$ is called a quasi-Einstein manifold if

$$
\operatorname{Ric}+\operatorname{Hess}(\rho)-\frac{1}{\tau} d \rho \otimes d \rho=\lambda g,
$$


where $\lambda$ is a constant. Thus, for $\tau=\infty$, a quasi-Einstein manifold is a gradient Ricci soliton (cf. [9]). For a quasi-Einstein manifold $(M, g, \rho)$ and an m-dimensional Riemannian manifold $\left(N, g_{1}\right)$, consider the warped product $\bar{M}=M \times_{u} N$ with the metric $\bar{g}=g+u^{2} g_{1}$, where

$$
u=e^{-\frac{\rho}{\tau}}
$$

is a positive function on $M$. Then, for $0<\tau<\infty$, we find

$$
\operatorname{grad} u=-\frac{u}{\tau} \operatorname{grad} \rho
$$

and therefore

$$
\operatorname{Hess}(u)=-\frac{u}{\tau} \operatorname{Hess}(\rho)+\frac{u}{\tau^{2}} d \rho \otimes d \rho
$$

Combining the above equation with (1), we obtain

$$
\operatorname{Hess}(u)=\frac{u}{\tau}(\operatorname{Ric}-\lambda g),
$$

which takes the form

$$
\frac{1}{2} £_{\xi} g+\alpha \operatorname{Ric}=\beta g,
$$

where $\xi=\operatorname{grad} u, \alpha=-\frac{u}{\tau}$ and $\beta=-\frac{\lambda u}{\tau}$. Note that for $\tau=m$, the warped product manifold $(\bar{M}, \bar{g})$ is an Einstein manifold if and only if $\left(N, g_{1}\right)$ is an Einstein manifold and the Ricci curvature of $(M, g)$ satisfies (1) (cf. [9]). Thus, choosing $\left(N, g_{1}\right)$ an Einstein manifold such that the warped product $(\bar{M}, \bar{g})$ is an Einstein manifold, we see, by Equation $(2)$, that $(g, \xi, \alpha, \beta)$ is a generalized Ricci soliton on the manifold $M$.

Consider now $(g, \xi, \alpha, \beta)$, a generalized Ricci soliton on an $n$-dimensional compact smooth manifold $M, n>2$, with $\xi$, a generalized geodesic vector field.

Denoting by $\eta$ the dual 1 -form of $\xi$, we can define the skew-symmetric $(1,1)$ tensor field $F$ by

$$
g(F X, Y)=\frac{1}{2}(d \eta)(X, Y)
$$

Then, we deduce

$$
\nabla_{X} \xi=\beta X-\alpha Q X+F X .
$$

As $\xi$ is a generalized geodesic vector field, we have

$$
\nabla_{\xi} \xi=f \xi,
$$

and taking the covariant derivative in the above equation, while using (3), we obtain

$$
\nabla_{X} \nabla_{\xi} \xi=X(f) \xi+f(\beta X-\alpha Q X+F X) .
$$

Additionally, we have

$$
\begin{aligned}
\nabla_{\xi} \nabla_{X} \xi & =\nabla_{\xi}(\beta X-\alpha Q X+F X) \\
& =\xi(\beta) X+\beta \nabla_{\xi} X-\xi(\alpha) Q X \\
& -\alpha\left(\nabla_{\xi} Q\right) X-\alpha Q\left(\nabla_{\xi} X\right)+\left(\nabla_{\xi} F\right) X+F\left(\nabla_{\xi} X\right)
\end{aligned}
$$

and

$$
\begin{aligned}
\nabla_{[X, \xi]} \xi= & \beta[X, \xi]-\alpha Q[X, \xi]+F[X, \xi] \\
= & \beta(\beta X-\alpha Q X+F X)-\beta \nabla_{\xi} X-\alpha Q(\beta X-\alpha Q X+F X)+\alpha Q\left(\nabla_{\xi} X\right) \\
& +F(\beta X-\alpha Q X+F X)-F\left(\nabla_{\xi} X\right) .
\end{aligned}
$$


Using (4) and the above two equations, we arrive at

$$
\begin{aligned}
R(X, \xi) \xi= & X(f) \xi+f(\beta X-\alpha Q X+F X)-\xi(\beta) X+\xi(\alpha) Q X+\alpha\left(\nabla_{\xi} Q\right) X \\
& -\left(\nabla_{\xi} F\right) X-\beta(\beta X-\alpha Q X+F X)+\alpha Q(\beta X-\alpha Q X+F X) \\
& -F(\beta X-\alpha Q X+F X) .
\end{aligned}
$$

Moreover, using (3), we have

$$
\begin{aligned}
R(X, Y) \xi= & X(\beta) Y-Y(\beta) X-X(\alpha) Q Y+Y(\alpha) Q X \\
& -\alpha\left(\nabla_{X} Q\right) Y+\alpha\left(\nabla_{Y} Q\right) X+\left(\nabla_{X} F\right) Y-\left(\nabla_{Y} F\right) X
\end{aligned}
$$

which gives

$$
\begin{aligned}
R(X, \xi) \xi= & X(\beta) \xi-\xi(\beta) X-X(\alpha) Q \xi+\xi(\alpha) Q X-\alpha\left(\nabla_{X} Q\right) \xi+\alpha\left(\nabla_{\xi} Q\right) X \\
& +\left(\nabla_{X} F\right) \xi-\left(\nabla_{\xi} F\right) X .
\end{aligned}
$$

Comparing the above equation with (4), we conclude

$$
\begin{aligned}
& X(\beta) \xi-X(\alpha) Q \xi-\alpha\left(\nabla_{X} Q\right) \xi+\left(\nabla_{X} F\right) \xi=X(f) \xi+f(\beta X-\alpha Q X+F X) \\
& -(\alpha Q-\beta I)^{2}(X)-2 \beta F X+\alpha Q F X+\alpha F Q X-F^{2} X .
\end{aligned}
$$

Taking $X=E_{i}$ in the above equation, for $\left\{E_{i}\right\}_{1 \leq i \leq n}$, an orthonormal frame field on $(M, g)$, and then taking the inner product with $E_{i}$ and summing the resulting equation, we obtain

$$
\begin{aligned}
& \xi(\beta)-\operatorname{Ric}(\xi, \operatorname{grad} \alpha)-\frac{\alpha}{2} \xi(r)-\sum_{i=1}^{n} g\left(\xi,\left(\nabla_{E_{i}} F\right) E_{i}\right) \\
& =\xi(f)+n \beta f-\alpha f r-\|\alpha Q-\beta I\|^{2}+\|F\|^{2} .
\end{aligned}
$$

Note that finding $\operatorname{div}(F \xi)$, yields the following

$$
\int_{M}\left(\|F\|^{2}+\sum_{i=1}^{n} g\left(\xi_{,}\left(\nabla_{E_{i}} F\right) E_{i}\right)\right)=0 .
$$

Now, integrating Equation (6) and using (7), we conclude

$$
\int_{M}\|\alpha Q-\beta I\|^{2}=\int_{M}\left(\xi(f)+n \beta f-\alpha f r-\xi(\beta)+\operatorname{Ric}(\xi, \operatorname{grad} \alpha)+\frac{\alpha}{2} \xi(r)\right) .
$$

Using $\operatorname{div}(f \xi)=\xi(f)+f(n \beta-\alpha r)$ and $\operatorname{div}(\beta \xi)=\xi(\beta)+\beta(n \beta-\alpha r)$ in the above equation, we obtain

$$
\int_{M}\|\alpha Q-\beta I\|^{2}=\int_{M}\left(\beta(n \beta-\alpha r)+\operatorname{Ric}(\xi, \operatorname{grad} \alpha)+\frac{\alpha}{2} \xi(r)\right) .
$$

By means of (5), we have

$$
\operatorname{Ric}(Y, \xi)=-(n-1) Y(\beta)-\operatorname{Ric}(Y, \operatorname{grad} \alpha)+r Y(\alpha)+\frac{\alpha}{2} Y(r)-\sum_{i=1}^{n} g\left(Y,\left(\nabla_{E_{i}} F\right) E_{i}\right) .
$$

Additionally, we have

$$
\operatorname{div}(F(\operatorname{grad} \alpha))=-\sum_{i=1}^{n} g\left(\operatorname{grad} \alpha,\left(\nabla_{E_{i}} F\right) E_{i}\right),
$$


where we used the symmetry of the Hessian operator $H_{\alpha}(X)=\nabla_{X} \operatorname{grad} \alpha$ and the skew symmetry of the operator $F$. Thus, Equation (9) implies

$$
\begin{aligned}
\operatorname{Ric}(\operatorname{grad} \alpha, \xi)= & -(n-1) g(\operatorname{grad} \alpha, \operatorname{grad} \beta)-\operatorname{Ric}(\operatorname{grad} \alpha, \operatorname{grad} \alpha)+r\|\operatorname{grad} \alpha\|^{2} \\
& +\frac{\alpha}{2} g(\operatorname{grad} \alpha, \operatorname{grad} r)+\operatorname{div}(F(\operatorname{grad} \alpha)) .
\end{aligned}
$$

Note that

$$
\begin{aligned}
\frac{\alpha}{2} \xi(r)= & \frac{1}{2} \operatorname{div}(r(\alpha \xi))-\frac{1}{2} r \operatorname{div}(\alpha \xi) \\
& =\frac{1}{2} \operatorname{div}(r(\alpha \xi))-\frac{1}{2} r(g(\xi, \operatorname{grad} \alpha)+\alpha(n \beta-\alpha r)) .
\end{aligned}
$$

Inserting Equations (10) and (11) into (8), we conclude

$$
\begin{aligned}
& \int_{M}\|\alpha Q-\beta I\|^{2}=\int_{M} \frac{1}{2}(n \beta-\alpha r)(2 \beta-\alpha r) \\
+ & \int_{M}\left(r\|\operatorname{grad} \alpha\|^{2}+g\left(\frac{\alpha}{2} \operatorname{grad} r-(n-1) \operatorname{grad} \beta-\frac{1}{2} r \xi, \operatorname{grad} \alpha\right)-\operatorname{Ric}(\operatorname{grad} \alpha, \operatorname{grad} \alpha)\right) .
\end{aligned}
$$

If the scalar curvature $r$ and the Ricci curvature Ric satisfy

$$
2 \beta<\alpha r \leq n \beta
$$

and

$$
\operatorname{Ric}(\operatorname{grad} \alpha, \operatorname{grad} \alpha) \geq r\|\operatorname{grad} \alpha\|^{2}+g\left(\frac{\alpha}{2} \operatorname{grad} r-(n-1) \operatorname{grad} \beta-\frac{1}{2} r \xi, \operatorname{grad} \alpha\right),
$$

then the above integral implies $\alpha Q=\beta I$, and this proves that

$$
£_{\xi} g=0,
$$

that is, the soliton is trivial. Thus, we proved Theorem 1 as presented below.

Theorem 1. If the potential field $\xi$ of a generalized Ricci soliton $(g, \xi, \alpha, \beta)$ on an $n$-dimensional compact smooth manifold $M(n>2)$ is a generalized geodesic vector field and the scalar and Ricci curvatures satisfy

$$
2 \beta<\alpha r \leq n \beta,
$$

$\operatorname{Ric}(\operatorname{grad} \alpha, \operatorname{grad} \alpha) \geq r\|\operatorname{grad} \alpha\|^{2}+g\left(\frac{\alpha}{2} \operatorname{grad} r-(n-1) \operatorname{grad} \beta-\frac{1}{2} r \xi, \operatorname{grad} \alpha\right)$, then the soliton is trivial.

As a consequence of Theorem 1 , for $\alpha=1$, we have the following:

Corollary 1. For an almost Ricci soliton $(g, \xi, \beta)$ on an $n$-dimensional compact smooth manifold $M(n>2)$ with a potential field, a generalized geodesic vector field is a trivial Ricci soliton if and only if the scalar curvature satisfies $2 \beta<\alpha r \leq n \beta$.

\section{Solitons with 2-Killing Vector Field}

Next, we put the restriction on the potential field $\xi$ of a generalized Ricci soliton $(g, \xi, \alpha, \beta)$ to be a 2-Killing vector field [10]. Recall that for a 2-Killing vector field, we have

$$
2 R(X, \xi ; \xi, Y)=g\left(\nabla_{X} \nabla_{\xi} \xi, Y\right)+g\left(\nabla_{Y} \nabla_{\xi} \xi, X\right)+2 g\left(\nabla_{X} \xi, \nabla_{Y} \xi\right), \quad X, Y \in \mathfrak{X}(M) .
$$


The above equation implies

$$
\operatorname{Ric}(\xi, \xi)=\operatorname{div}\left(\nabla_{\xi} \xi\right)+\|\nabla \xi\|^{2},
$$

which integrates into

$$
\int_{M}\left(\operatorname{Ric}(\xi, \xi)-\|\nabla \xi\|^{2}\right)=0 .
$$

Now, using (3), we obtain

$$
\begin{aligned}
\|\nabla \xi\|^{2} & =n \beta^{2}+\alpha^{2}\|Q\|^{2}+\|F\|^{2}-2 \alpha \beta r \\
& =\alpha^{2}\left(\|Q\|^{2}-\frac{1}{n} r^{2}\right)+\frac{1}{n}(n \beta-\alpha r)^{2}+\|F\|^{2} .
\end{aligned}
$$

Inserting the above equation into (12), we have

$$
\int_{M} \alpha^{2}\left(\|Q\|^{2}-\frac{1}{n} r^{2}\right)=\int_{M}\left(\operatorname{Ric}(\xi, \xi)-\frac{1}{n}(n \beta-\alpha r)^{2}-\|F\|^{2}\right) .
$$

Using Equation (9), we obtain

$$
\operatorname{Ric}(\xi, \xi)=-(n-1) \xi(\beta)-\operatorname{Ric}(\xi, \operatorname{grad} \alpha)+r \xi(\alpha)+\frac{\alpha}{2} \xi(r)-\sum_{i=1}^{n} g\left(\xi_{,}\left(\nabla_{E_{i}} F\right) E_{i}\right)
$$

and using $\alpha \xi(r)=\xi(\alpha r)-r \xi(\alpha)$, we conclude

$$
\operatorname{Ric}(\xi, \xi)=-\xi(n \beta-\alpha r)-\operatorname{Ric}(\xi, \operatorname{grad} \alpha)-\frac{\alpha}{2} \xi(r)+\xi(\beta)-\sum_{i=1}^{n} g\left(\xi,\left(\nabla_{E_{i}} F\right) E_{i}\right) .
$$

Note that we have $\xi(n \beta-\alpha r)=\operatorname{div}((n \beta-\alpha r) \xi)-(n \beta-\alpha r)^{2}$, and inserting it in the above equation and integrating it, we arrive at

$$
\int_{M} \operatorname{Ric}(\xi, \xi)=\int_{M}\left((n \beta-\alpha r)^{2}-\operatorname{Ric}(\xi, \operatorname{grad} \alpha)-\frac{\alpha}{2} \xi(r)+\xi(\beta)-\sum_{i=1}^{n} g\left(\xi,\left(\nabla_{E_{i}} F\right) E_{i}\right)\right) .
$$

Now, using (7) with the above equation, we have

$$
\int_{M}\left(\operatorname{Ric}(\xi, \xi)-\|F\|^{2}\right)=\int_{M}\left((n \beta-\alpha r)^{2}-\operatorname{Ric}(\xi, \operatorname{grad} \alpha)-\frac{\alpha}{2} \xi(r)+\xi(\beta)\right) .
$$

Moreover, we have $\xi(\beta)=\operatorname{div}(\beta \xi)-\beta(n \beta-\alpha r)$ and $\alpha \xi(r)=\xi(\alpha r)-r \xi(\alpha)=$ $\operatorname{div}(\alpha r)-\alpha r(n \beta-\alpha r)-r \xi(\alpha)$, and combining these equations, we have

$$
\begin{aligned}
-\frac{\alpha}{2} \xi(r)+\xi(\beta) & =\operatorname{div}\left(\beta-\frac{1}{2} \alpha r\right)+\frac{1}{2} \alpha r(n \beta-\alpha r)-\beta(n \beta-\alpha r)+\frac{1}{2} r \xi(\alpha) \\
& =\operatorname{div}\left(\beta-\frac{1}{2} \alpha r\right)+(n \beta-\alpha r)\left(\frac{1}{2} \alpha r-\beta\right)+\frac{1}{2} r \xi(\alpha) .
\end{aligned}
$$

Inserting the above equation into (14), we arrive at

$$
\begin{aligned}
\int_{M}(\operatorname{Ric}(\xi, \xi)- & \left.\|F\|^{2}\right) \\
& =\int_{M}\left((n \beta-\alpha r)^{2}-\operatorname{Ric}(\xi, \operatorname{grad} \alpha)+(n \beta-\alpha r)\left(\frac{1}{2} \alpha r-\beta\right)+\frac{1}{2} r \xi(\alpha)\right) .
\end{aligned}
$$


Combining the above equation with (13), we obtain

$$
\begin{aligned}
& \int_{M} \alpha^{2}\left(\|Q\|^{2}-\frac{1}{n} r^{2}\right) \\
& \quad=\int_{M}\left(\frac{n-1}{n}(n \beta-\alpha r)^{2}-\operatorname{Ric}(\xi, \operatorname{grad} \alpha)+(n \beta-\alpha r)\left(\frac{1}{2} \alpha r-\beta\right)+\frac{1}{2} r \xi(\alpha)\right) .
\end{aligned}
$$

Inserting Equation (10) into the above equation, we conclude

$$
\begin{aligned}
& \int_{M} \alpha^{2}\left(\|Q\|^{2}-\frac{1}{n} r^{2}\right)=\int_{M} \operatorname{Ric}(\operatorname{grad} \alpha, \operatorname{grad} \alpha)+\frac{n-2}{2 n} \int_{M}(n \beta-\alpha r)(2 n \beta-\alpha r) \\
& -\int_{M}\left(r\|\operatorname{grad} \alpha\|^{2}-g\left(\frac{r}{2} \xi-\frac{\alpha}{2} \operatorname{grad} r+(n-1) \operatorname{grad} \beta, \operatorname{grad} \alpha\right)\right) .
\end{aligned}
$$

If the scalar curvature $r$ and the Ricci curvature Ric satisfy

$$
n \beta \leq \alpha r \leq 2 n \beta
$$

$\operatorname{Ric}(\operatorname{grad} \alpha, \operatorname{grad} \alpha) \leq r\|\operatorname{grad} \alpha\|^{2}-g\left(\frac{r}{2} \xi-\frac{\alpha}{2} \operatorname{grad} r+(n-1) \operatorname{grad} \beta, \operatorname{grad} \alpha\right)$, then the above integral implies

$$
\alpha^{2}\left(\|Q\|^{2}-\frac{1}{n} r^{2}\right)=0
$$

If $\alpha=0$, then Equation (15) implies

$$
n(n-2) \int_{M} \beta^{2}=0,
$$

which, in view of $n>2$, gives $\beta=0$, and, consequently, in this case, we obtain $£_{\xi g}=0$; that is, the soliton is trivial. Note that by Schwart's inequality, we have $\|Q\|^{2} \geq \frac{1}{n} r^{2}$, and equality holds if and only if $Q=\frac{r}{n} I$. Thus, if $\|Q\|^{2}-\frac{1}{n} r^{2}=0$, then we conclude $Q=\frac{r}{n} I$ and using the definition of soliton, we have

$$
£_{\xi} g=\frac{2}{n}(n \beta-\alpha r) g
$$

Using the fact that $\xi$ is 2-Killing, in the above equation, we obtain

$$
\begin{aligned}
0 & =£_{\xi} £_{\xi} g=\frac{2}{n}(\xi(n \beta-\alpha r)) g+\frac{2}{n}(n \beta-\alpha r) £_{\xi} g \\
& =\left(\xi(n \beta-\alpha r)+\frac{2}{n}(n \beta-\alpha r)^{2}\right) g
\end{aligned}
$$

that is, $\xi(n \beta-\alpha r)+\frac{2}{n}(n \beta-\alpha r)^{2}=0$. Integrating this equation while using $\xi(n \beta-\alpha r)=$ $\operatorname{div}((n \beta-\alpha r) \xi)-(n \beta-\alpha r)^{2}$, we conclude

$$
\frac{n-2}{n} \int_{M}(n \beta-\alpha r)^{2}=0 .
$$

This proves that $n \beta-\alpha r=0$, and, consequently, Equation (16) yields

$$
£_{\xi} g=0,
$$


that is, the soliton is trivial. Hence, we have the theorem provided below.

Theorem 2. If the potential field $\xi$ of a generalized Ricci soliton $(g, \xi, \alpha, \beta)$ on an n-dimensional compact smooth manifold $M(n>2)$ is a 2-Killing vector field and the scalar and Ricci curvatures satisfy

$$
\begin{gathered}
n \beta<\alpha r \leq 2 n \beta, \\
\operatorname{Ric}(\operatorname{grad} \alpha, \operatorname{grad} \alpha) \leq r\|\operatorname{grad} \alpha\|^{2}-g\left(\frac{r}{2} \xi-\frac{\alpha}{2} \operatorname{grad} r+(n-1) \operatorname{grad} \beta, \operatorname{grad} \alpha\right),
\end{gathered}
$$

then the soliton is trivial.

As a consequence of Theorem 2, for $\alpha=1$, we have the following corollary (with the converse implication also being true).

Corollary 2. The potential field $\xi$ of an almost Ricci soliton $(g, \xi, \beta)$ on an $n$-dimensional compact smooth manifold $M(n>2)$ is a 2-Killing vector field and the scalar curvature $r$ satisfies $n \beta<r \leq$ $2 n \beta$ if and only if $(g, \xi, \beta)$ is a trivial Ricci soliton.

\section{Conclusions}

In this paper, we studied generalized Ricci solitons $(g, \xi, \alpha, \beta)$ on an $n$-dimensional compact smooth manifold $M$ for $n>2$ by restricting the as-generalized geodesic vector field. It was shown that with suitable bounds on the scalar curvature, the Ricci curvature in the direction of $\operatorname{grad}(\alpha)$ was rendered trivial (cf. Theorem 1), and this result, as a particular case, also provides a characterization of trivial Ricci soliton in terms of an almost Ricci soliton. Furthermore, by restricting $\xi$ to a 2-Killing vector field, it was shown that suitable bounds on the scalar curvature make the Ricci curvature in the direction of $\operatorname{grad}(\alpha)$ a trivial soliton (cf. Theorem 2), and as a particular case, we obtained characterization of the trivial Ricci soliton via an almost Ricci soliton using 2-Killing vector fields.

This study marks the beginning of the generalized solitons $(g, \xi, \alpha, \beta)$ on an $n$-dimensional compact smooth manifold $M$, and the results to date use only the restrictions on the potential field $\xi$. It will be interesting to determine what restrictions should be used on the functions $\alpha$ and $\beta$ to obtain conclusions similar to those of Theorems 1 and 2.

Author Contributions: Formal analysis, S.D.; Funding acquisition, A.I.; Investigation, A.M.B. All authors have read and agreed to the published version of the manuscript.

Funding: This research received no external funding.

Institutional Review Board Statement: Not applicable.

Informed Consent Statement: Not applicable.

Data Availability Statement: Not applicable.

Acknowledgments: This work is supported by Taif University Researchers Supporting Project number (TURSP-2020/223), Taif University, Taif, Saudi Arabia.

Conflicts of Interest: The authors declare that they have no conflict of interest.

\section{References}

1. Hamilton, R.S. The Ricci flow on surfaces. Contemp. Math. 1988, 71, 237-261. [CrossRef]

2. Hirică, I.E.; Udrişte, C. Ricci and Riemann solitons. Balkan J. Geom. Appl. 2016, 21, 35-44.

3. Németh, S.Z. Five kinds of monotone vector fields. Pure Math. Appl. 1998, 9, 417-428.

4. Cruz Neto, J.X.; Melo, I.D.; Sousa, P.A. Non-existence of strictly monotone vector fields on certain Riemannian manifolds. Acta Math. Hungar. 2015, 146, 240-246. [CrossRef]

5. Chen, B.-Y.; Deshmukh, S. A note on Yamabe solitons. Balkan J. Geom. Appl. 2018, 23, 37-43.

6. Deshmukh, S.; Peska, P.; Bin Turki, N. Geodesic Vector Fields on a Riemannian Manifold. Mathematics 2020, 8, 137. [CrossRef]

7. Choquet-Bruhat, Y.; DeWitt-Morette, C.; Dillard-Bleichi, M. Analysis, Manifolds and Physics; North-Holland: Amsterdam, The Netherlands, 1977.

8. Fialkow, A. Conformal geodesics. Trans. Am. Math. Soc. 1939, 45, 443-473. [CrossRef] 
9. Wang, L.F. On noncompact $\tau$-quasi-Einstein metrics. Pacific J. Math. 2011, 254, 449-464. [CrossRef]

10. Oprea, T. 2-Killing vector fields on Riemannian manifolds. Balkan J. Geom. Appl. 2008, 13, 87-92. 